\title{
Does Hawking predict the correct temperature of a black-hole?
}

https://doi.org/10.1515/astro-2020-0008

Received Nov 14, 2019; accepted May 05, 2020

Abstract: In our study of the validity of Hawking's predicted radiation temperature of a black-hole, we found that the calculated temperature is another form of Zeldovich's expression for the cosmological constant. We reasoned that as Zeldovich predicted the extreme value of cosmological constant thus Hawking might have also predicted an extreme temperature. However, the actual temperature might be something different. This result implies that all predictions based on Hawking's radiation temperature might be incorrect.

Keywords: Hawking temperature, cosmological constant, quantum-gravitational effect, black-hole evaporations and explosions

\section{Introduction}

The Hawking radiation temperature is a fascinating quantum effect finding in black-hole physics. The existence of this temperature has been proposed by Hawking while studying black hole thermodynamics (Hawking 1975). For the first time it seemed that, contrary to classical black-hole, quantum black-holes emit particles due to some quantum effect at the event horizon (Hawking 1974). Hawking radiation occurs when one of a pair of quantum particle falls into a black-hole's edge while the other escapes, resulting in Hawking radiation. Applying this idea, he estimated the time scale when a black-hole completely evaporated and suggesting the possibility of an explosion of a black-hole when the gravity of the black-hole weakened itself due to mass loss by particle creation phenomenon (Hawking 1974).

We are concerned that no experimental set up to measure this temperature quantitatively exists so far to validate this theory and its other predictions. There have been some attempts to create the analogous black-hole in a laboratory setting but many issues remain (Unruh \& Schüzhold 2012; Steinhauer 2014). However, it's pretty difficult to be confident whether his predicted temperature is precisely correct or not.

Corresponding Author: Kapil Chandra: Department of physics, University of Bastar, Dharampura 1, Jagdalpur [CG], India, PIN - 494226; Email: kapil.chandra@gov.in

\section{A new approach to re-derive the mathematical expression of Hawking's temperature}

To address this open question, we studied this temperature by re-deriving the mathematical expression for temperature from other established theory of fundamental physics. We also correlated this with existing theories. After deriving the mathematical expression of this temperature we compared it with experimentally observed results.

To derive the desired expression for Hawking radiation temperature we write following balance equation between Planck force which are either function of mass or space parameter (Planck 1899),

$$
\frac{h c}{R^{2}}=\frac{m^{2} c^{3}}{h}
$$

All constants have their usual meanings and the mass $m$ and space parameter $R$ are the only variables introduced here. Both equations are interrelated via Compton relation.

It is noteworthy that both sides of Eq. (1) denote a quantum of force; either as a function of distance or a function of mass. These forces are due to quantization of space/mass. We believe that this expression can be used to estimate the theoretical value of strong force or nuclear force by replacing the $m$ as mass of proton and $R$ as size of nucleus respectively. We are not going in its detail, it's beyond the scope of this article.

We know that the Hawking radiation temperature is due to quantum - gravitational effect. This has been suggested by Hawking himself and its mathematical expres-

¿ Open Access. (c) 2020 K. Chandra et al., published by De Gruyter. (cc) BY 4.0 License

This work is licensed under the Creative Commons Attribution 
sion also expresses that since it contains both Planck and universal gravitational constant. We observe from Eq. (1) the balance of force has only the Planck constant i.e. it is in quantum terms only. However, to get the quantum - gravitational term we introduce the quantum - gravitational effect there by substituting the following classical relation between the mass and space parameter as written in the form

$$
R=\frac{G m}{c^{2}}
$$

We view this as a pure classical relation between mass and space since these variables are proportional to the universal gravitational constant. If we now substitute the Eq. (2) into Eq. (1) we get the following,

$$
\frac{h c^{5}}{G^{2} m^{2}}=\frac{R^{2} c^{7}}{G^{2} h}
$$

This expression is a quantum - gravitational relationship since it incorporates both the Planck constant and the gravitational constant (Chandra 2019).

\section{Interrelation between Hawking temperature and cosmological constant}

The first term corresponds to Hawking's radiation temperature. It can be seen by letting energy $E=F . R$ where $R$ is expression in Eq. (2) and letting $E=k_{B} T$ where $k_{B}$ is Boltzmann constant; the temperature $T$ will be,

$$
T=\frac{h c^{3}}{G m k_{B}}
$$

This is Hawking's expression for temperature without a numerical factor $1 / 16 \pi^{2}$.

Similarly if we let $E=F . R$ the RHS of Eq. (3) gives us energy density of space as followings,

$$
\rho_{E}=\frac{c^{7}}{G^{2} h}
$$

This expression was derived by Ya B. Zeldovich where he assumed that quantum fluctuation of the vacuum give rise to the vacuum energy density or cosmological constant (Zeldovich 1967).

This description clarifies that the mathematical expression of Hawking temperature corresponds to the Zeldovich's expression of vacuum energy density or cosmological constant theoretically since both physical entity originate via similar mechanism: the quantum - gravitational effect. It implies that the Hawking temperature and cosmological constant are closely related and, in fact, equivalent to each other; this can be seen from Eq. (3), if we compare both entity it reduce to well-known Compton equation. It also reveals that the Hawking radiation temperature is due to quantization of mass while cosmological constant is due to quantization of space, Hawking's and Zeldovich's formulas only differ in their mathematical forms.

However, the intriguing fact is that Zeldovich's formula estimates a much larger numerical value of cosmological constant than its observed value (Weinberg 1989). Thus, we reasoned that Hawking might also be estimating its large value but the observed temperature might be something different, in fact, it must be much smaller with respect to the predicted one.

This result hints that all the predictions which are based on the numerical value of Hawking temperature must be altered such as the black-hole evaporation time (Hawking 1974).

\section{Conclusions}

In summary, we have shown that Hawking's extreme temperature of a black-hole must be much smaller than predicted because his derived expression corresponds to the Zeldovich's expression for vacuum energy density what produced the cosmological constant problem.

Since Hawking's radiation temperature is not measured by any experiment it's not easy to say our conclusion is correct. Also we can't really say what would be the precise temperature of a black-hole. The answer to the cosmological constant problem may yield the precise value of Hawking temperature problem. Further research recommended in this area.

Acknowledgment: I pay my hearty gratitude To SE Karram for his support to prepare this manuscript and fruitful discussions.

\section{References}

Chandra K. 2019. Why Zeldovich failed to estimate the precise value of cosmological constant in Planck unit? Journal of High Energy Physics. Gravitation Cosmol. 5(04):1098-1104.

Hawking SW. 1975. Particle creation by black holes. Commun Math Phys. 43(3):199-220.

Hawking SW. 1974. Black hole explosions? Nature. 248(5443):3031.

Planck M. 1899. Preusse. Aka. Wiss; 479-480 p.

Steinhauer J. 2014. Observation of self-amplifying Hawking radiation in an analogue black-hole laser. Nat Phys. 10(11):864-869. 
Unruh WG, Schützhold R. 2012. Hawking radiation from "phase horizons" in laser filaments? Phys Rev D Part Fields Gravit Cosmol. 86(6):064006.

Weinberg S. 1989. The cosmological constant problme. Rev Mod Phys. 61(1):1-23.
Zeldovich YB. 1968. The cosmological constant and the theory of elementary particles. Sov Phys Usp. 11(3):381-393. 